\title{
Overexpression of EPHB4 Is Associated with Poor Survival of Patients with Gastric Cancer
}

\author{
JIE YIN $^{1,2,3^{*}}$, YUXIN CUI $^{1 *}$, LITING LI $^{1,4}$, JIAFU JI $^{4}$ and WEN G. JIANG ${ }^{1}$ \\ ${ }^{1}$ Cardiff China Medical Research Collaborative, Cardiff University School of Medicine, Cardiff, U.K.; \\ ${ }^{2}$ Department of General Surgery, Beijing Friendship Hospital, Capital Medical University, Beijing, P.R. China; \\ ${ }^{3}$ Beijing Key Laboratory of Cancer Invasion and Metastasis, \\ Research \& National Clinical Research Center for Digestive Diseases, Beijing, P.R. China; \\ ${ }^{4}$ Department of Gastrointestinal Surgery, Beijing Cancer Hospital and Key Laboratory of Carcinogenesis and \\ Translational Research, Peking University Cancer Hospital and Institute, Beijing, P.R. China
}

\begin{abstract}
Background: Increased expression of erythropoietinproducing human hepatoma (EPHB4) leads to enhanced cell migration, growth and adhesion in tumor cells. However, little is known regarding the effects of EPHB4 in gastric cancer. The present study aimed to examine the clinical relevance of EPHB4 and its association with the prognosis of gastric cancer. Materials and Methods: EPHB4 transcript expression in 324 gastric cancer samples with paired adjacent normal gastric tissues was determined using quantitative polymerase chain reaction and the results were statistically analyzed against patient clinicopathological data. AGS and HGC27 cell lines were transfected with EPHB4 siRNA and the effects examined by functional analysis. Results: EPHB4 mRNA levels in gastric cancer tissues were significantly elevated when compared to non-cancerous tissues $(p=0.0110)$. Tissue samples from male patients exhibited lower expression than those from female patients ( $p=0.0110)$. Non-cardiac gastric tumors (fundus, corpus and pylorus) expressed a higher number of EPHB4 transcripts in comparison to cardiac gastric tumors $(p<0.001)$. Increased expression of EPHB4 was significantly associated with poorer overall $(p=0.0051)$ and progression-free ( $p=0.0262$ ) survival. EPHB4 knockdown appeared to reduce post-wound migration of AGS cells $(p=0.0057)$ and increase migration of HGC27 cells ( $p=0.0337)$. EPHB4 knockdown significantly increased adhesive ability in HGC27 $(p<0.0001)$. Conclusion: The expression of EPHB4 was increased in gastric cancer and increased EPHB4 expression was correlated with
\end{abstract}

*These Authors contributed equally to this work.

Correspondence to: Professor Wen G. Jiang, Cardiff China Medical Research Collaborative, School of Medicine, Cardiff University, Heath Park, Cardiff CF14 4XN, U.K. E-mail: jiangw@cardiff.ac.uk

Key Words: EPHB4, gastric cancer, survival. poor survival. Knockdown of EPHB4 promoted adhesion and exerted diverse effects on migration of gastric cancer cells. Further investigations may highlight its predictive and therapeutic potential in gastric cancer.

The erythropoietin-producing human hepatoma (EPH) family is the largest among transmembrane receptor tyrosine kinases (RTKs), and consists of 16 members divided into two groups: EPHA (EPHA1-10) and EPHB (EPHB1-6). The EPH receptors and their membrane-anchored ephrin ligands regulate tumorigenesis and angiogenesis (1). EPH signaling triggers multiple pathways, most notably the RAS (R-RAS, H-RAS, N-RAS) and RHO (RHO, RAC1, CDC42) family of small GTPases, making it critical to many cellular functions including cellular proliferation, cytoskeletal dynamics and cell adhesion (2, 3). EPHB receptors have a single transmembrane domain and a short cytoplasmic tail and usually recognize transmembrane EPHB ligands (4).

Among the EPHB receptors, EPHB4 has been extensively studied in various tumor types. Increased expression of EPHB4 has been reported in several advanced cancer types, including bladder (5), colon (6), prostate (7), gastroesophageal junction (8), breast (9), non-small cell lung carcinoma (10), and mesothelioma (11), but with low or no expression in most normal tissues.

In order to elucidate the roles of EPHB4 in gastric cancer, we examined the expression of $E P H B 4$ in normal and surgical gastric cancer specimens, and also in gastric cancer cell lines. We analyzed the association between EPHB4 expression and overall (OS) and progression-free (PFS) survival.

\section{Materials and Methods}

Cell lines and culture conditions. Human gastric cancer cell lines AGS and HGC27 were purchased from the European Collection of Cell Cultures (Salisbury, UK) and incubated at $37^{\circ} \mathrm{C}$, with $5 \% \mathrm{CO}_{2}$ and $95 \%$ humidity. These cell lines were maintained in Dulbecco's 
Table I. Primers used for quantitative polymerase chain reaction.

\begin{tabular}{lll}
\hline Primer & Forward primer & Reverse primer \\
\hline$E P H B 4-1$ & CATCGGACATGGTACTAAGGA & ACTGAACCTGACCGTACATTTTGCAAATTCCCTCACAG \\
$E P H B 4-2$ & GGAACATCACAGCCAGAC & ACTGAACCTGACCGTACAAGGACCACACCCACGACT \\
GAPDH & AAGGTCATCCATGACAACTT & ACTGAACCTGACCGTACAAGCCATCCACAGTCTTCTG \\
\hline
\end{tabular}

EPHB4, Erythropoietin-producing human hepatoma B4; GAPDH, glyceraldehyde-3-phosphate dehydrogenase.

modified Eagle's medium (DMEM) supplemented with $10 \%$ fetal calf serum (PAA Laboratories Ltd., Somerset, UK), penicillin, streptomycin and amphotericin B.

Human gastric tissues. Gastric adenocarcinoma and Siewert type III gastro-oesophageal junction adenocarcinoma tissues $(n=245)$ with matched adjacent background tissues $(n=158)$ were immediately collected after surgical resection at the Beijing Cancer Hospital with informed consent from the patients. All patients underwent surgery without any prior treatment. The tissue samples were stored at $-80^{\circ} \mathrm{C}$ at the Tissue Bank of Peking University Oncology School with a record of the relevant clinical and histopathological data. All protocols were reviewed and approved by the Beijing Cancer Hospital Research Ethics Committee (MTA10062009).

$R N A$ isolation and quantitative real-time polymerase chain reaction $(q P C R)$. RNA was extracted from confluent cells in a $25 \mathrm{~cm}^{2}$ flask using total RNA isolation (TRI) reagent (Sigma-Aldrich, Dorset, UK). Fresh frozen tissues were also first homogenized in the TRI reagent. First strand cDNA was synthesized from $1 \mu \mathrm{g}$ RNA using a first-strand DNA synthesis kit (Bio-Rad, Hemel Hempstead, UK). Quantitative analysis of EPHB4 mRNA expression in gastric cancer tissues was performed using Amplifluor ${ }^{\mathrm{TM}}$-based real-time PCR, in which a 6-carboxy-fluorescine-tagged Uniprimer ${ }^{\mathrm{TM}}$ (Biosearch Technologies, Inc., Petaluma, CA, USA.) was used as a probe along with a pair of specific primers with an addition of a Z-sequence (actgaacctgaccgtaca) to the 5'-end of the reverse primer. The quality of cDNA samples was verified using glyceraldehyde-3-phosphate dehydrogenase $(G A P D H)$ as a housekeeping gene. All the primer sequences used are listed in Table I.

Quantitative analyses of transcript expression of EPHB4 in human gastric cancer. Following the real-time PCR quantification of each gene transcript, the number of samples with valid data for each individual gene was: 324 samples for EPHB4. This cohort comprised 231 men $(71.3 \%)$ and 93 women $(28.7 \%)$. Data are shown in Table II.

Kaplan-Meier survival analysis. The association between EPHB4 expression and survival of patients with gastric cancer was assessed using an online Kaplan-Meier survival analysis tool (http://kmplot.com) $(12,13)$. The tool allowed us to analyze the OS from 876 cases of gastric cancer and PFS from 641 cases which were subjected to expression profiling using Affymetrix GeneChip microarray (EPHB4 Probeset ID: 202894_at).

Transfection with EPHB4 siRNA. Short interfering RNAs (siRNA) specific for EPHB4 and the no template control (NTC-siRNA) were obtained from Dharmacon (GE Healthcare, London, UK). AGS and
HGC27 cells were transfected with $100 \mathrm{nM}$ of the siRNA reagents in serum-free DMEM using Lipofectamine ${ }^{\mathrm{TM}} 3000$ Transfection Reagent (Invitrogen, Carlsbad, CA, USA) according to the manufacturer's instructions. After transfection for $20 \mathrm{~h}$, the medium was replaced with complete culture medium. Cells were analyzed at optimal time-points by different assays.

In vitro cell proliferation assay. Cells were seeded into 96-well plates in complete growth culture medium at a density of 5,000 cells/well. The cells were incubated over a period of up to 1 day, and were then fixed with $4 \%$ formaldehyde followed by staining with $0.5 \%$ crystal violet. The crystal violet was dissolved in $10 \%$ acetic acid prior to a colorimetric detection of cell density at a wavelength of $580 \mathrm{~nm}$ using the ELx800 spectrophotometer to test transfection after 24 and $48 \mathrm{~h}$.

Cell-matrix adhesion assay. The 96-well culture plate was precoated with $5 \mu \mathrm{g} / \mathrm{well}$ of Matrigel (BD Biosciences, San Diego, CA, USA) and air-dried. Following the rehydration, 20,000 cells were seeded in each well. After an incubation of $40 \mathrm{~min}$, non-adherent cells were washed-off using phosphate-buffered saline. The adherent cells were counted after fixation and staining using crystal violet.

Wound-healing assay. The cells were cultivated until they reached confluency. A scrape in the cell monolayer was made in one direction with a fine gauge needle. The wounded cell monolayers were washed with phosphate-buffered saline to remove cell debris. The gap remaining at different time points were analyzed using an inverted microscope and indicated the wound-healing migration of the cells.

Adhesion and migration measurement using electric cell substrate impedance sensing (ECIS) analysis. ECIS 9600 system (Applied Biophysics Inc., Troy, NY, USA) was used to monitor the spreading, attachment and migratory behavior of gastric cancer cells. Briefly, AGS and HGC27 cells were seeded onto ECIS 96W1E arrays and adhesion of cells to the culture surface between the two electrodes was monitored via measuring electrical resistance. Once a confluent monolayer had been formed, the cells were damaged by applying an electrical current $(2600 \mu \mathrm{A}, 60 \mathrm{kHz})$ for $20 \mathrm{~s}$ to create a break in the cell monolayer. The rate of change in impedance as cells migrated back onto the electrode was subsequently monitored and analyzed.

Statistical analysis. Statistical analyses were performed using SPSS (version 11; SPSS, Inc., Chicago, IL, USA). Mann-Whitney $U$-test and $t$-test were used for non-parametric and normally distributed data, respectively, including research data from the clinical cohort and cell-based experiments. Differences were considered to be statistically significant at $p<0.05$. 
Table II. Gene expression of erythropoietin-producing human hepatoma (EPHB4) in the gastric cancer cohort.

\begin{tabular}{|c|c|c|c|c|c|c|c|}
\hline \multirow[b]{2}{*}{ Variable } & & \multirow[b]{2}{*}{ Number } & \multicolumn{5}{|c|}{$E P H B 4$ expression } \\
\hline & & & Number with data missing & Mean & SD & SEM & $\mathrm{p}$-Value \\
\hline \multirow[t]{2}{*}{ Tissue } & Tumour & 180 & 144 & 170,052 & 773,731 & 57,671 & \\
\hline & Adjacent normal & 100 & 224 & 20,684 & 33,995 & 3,399 & 0.0110 \\
\hline \multirow[t]{2}{*}{ Gender } & Male & 132 & 99 & 157,874 & 632,492 & 55,051 & \\
\hline & Female & 48 & 45 & 203,543 & $1,078,620$ & 155,685 & 0.0110 \\
\hline \multirow[t]{2}{*}{ Gastric location } & Non-cardiac & 138 & 119 & 187,024 & 873,644 & 74,370 & \\
\hline & Cardiac & 34 & 18 & 82,278 & 92,903 & 15,933 & $<0.0010$ \\
\hline \multirow[t]{6}{*}{ T-Stage } & $\mathrm{T} 1$ & 9 & 7 & 75,569 & 95,604 & 31,868 & \\
\hline & $\mathrm{T} 2$ & 11 & 15 & 59,493 & 65,271 & 19,680 & 0.6700 \\
\hline & $\mathrm{T} 3$ & 29 & 12 & 70,894 & 101,146 & 18,782 & 0.9000 \\
\hline & $\mathrm{T} 4$ & 126 & 107 & 215,073 & 920,410 & 81,997 & 0.1200 \\
\hline & $\mathrm{T} 1+\mathrm{T} 2$ & 20 & 22 & 66,727 & 78,473 & 17,547 & \\
\hline & $\mathrm{T} 3+\mathrm{T} 4$ & 155 & 119 & 188,098 & 832,267 & 66,849 & 0.0810 \\
\hline \multirow[t]{5}{*}{ N-Stage } & No & 35 & 36 & 78,403 & 89,146 & 15,068 & \\
\hline & N1 & 22 & 26 & 108,561 & 174,811 & 37,270 & 0.4600 \\
\hline & $\mathrm{N} 2$ & 40 & 25 & 79,139 & 93,247 & 14,744 & 0.9700 \\
\hline & N3 & 80 & 54 & 278,016 & $1,148,492$ & 128,405 & 0.1300 \\
\hline & $\mathrm{N} 1+\mathrm{N} 2+\mathrm{N} 3$ & 142 & 105 & 195,741 & 868,834 & 72,911 & 0.1200 \\
\hline \multirow[t]{2}{*}{ M-Stage } & M0 & 152 & 130 & 189,784 & 840,451 & 68,170 & \\
\hline & M1 & 27 & 14 & 64,868 & 67,037 & 12,901 & 0.0740 \\
\hline \multirow[t]{6}{*}{ TNM } & TNM1 & 14 & 11 & 77,954 & 88,251 & 23,586 & \\
\hline & TNM2 & 26 & 34 & 80,552 & 90,395 & 17,728 & 0.9300 \\
\hline & TNM3 & 129 & 92 & 209,423 & 910,510 & 80,166 & 0.1200 \\
\hline & TNM4 & 5 & 4 & 55,532 & 56,286 & 25,172 & 0.5300 \\
\hline & TNM1+2 & 40 & 45 & 79,643 & 88,519 & 13,996 & \\
\hline & TNM3+4 & 134 & 96 & 203,680 & 893,765 & 77,210 & 0.1200 \\
\hline \multirow[t]{4}{*}{ Differentiation } & High-moderate & 5 & 1 & 198,744 & 170,626 & 76,306 & \\
\hline & Moderate & 32 & 30 & 112,818 & 168,790 & 29,838 & 0.3400 \\
\hline & Moderate-low & 43 & 39 & 92,000 & 100,872 & 15,383 & 0.2400 \\
\hline & Low & 74 & 64 & 276,565 & $1,193,693$ & 138,764 & 0.6300 \\
\hline \multirow[t]{2}{*}{ Embolus } & Without & 79 & 74 & 77,534 & 117,792 & 13,253 & \\
\hline & With & 92 & 64 & 262,395 & $1,071,379$ & 111,699 & 0.1000 \\
\hline \multirow[t]{2}{*}{ Surgery } & Non-radical & 43 & 28 & 100,748 & 234,366 & 35,740 & \\
\hline & Radical & 136 & 113 & 193,136 & 880,020 & 75,461 & 0.2700 \\
\hline
\end{tabular}

SD: Standard deviation; SEM: standard error of mean; T: stage of tumour; N: stage of lymph node; M: metastasis.

\section{Results}

Increased expression of EPHB4 in human gastric cancer. Transcript levels of EPHB4 were determined in the gastric cancer cohort using real-time PCR. The results showed that EPHB4 expression was also significantly up-regulated in gastric tumors compared to normal tissues $(p=0.0110)$ (Table II). Differential expression of EPHB4 in gastric cancer was associated with gender, samples from males exhibiting lower expression than those from females $(p=0.0110)$. According to the tumor location, non-cardiac gastric tumors expressed higher levels of $E P H B 4$ transcript in comparison to tumors of the gastric cardia $(p<0.001)$. Although the transcript levels of $E P H B 4$ in those with $\mathrm{TNM} 3+4$ and $\mathrm{N} 1+2+3$ tumors were significantly higher than those of the group with TNM1+2 and N0 tumor, there was no statistically significant difference $(p=0.1200$ and $p=0.1200)$. Similarly, no association was observed with local invasion, lymph node involvement or distant metastases.

Association of EPHB4 expression with the survival of patients with gastric cancer. The Kaplan-Meier survival analysis showed that the elevated expression of EPHB4 was significantly associated with poorer OS $(p=0.0051)$, given a cutoff value of 537 in expression (range=81-6,435) of EPHB4 (Figure 1A). Similarly, increased EPHB4 expression was also associated with poor PFS $(p=0.0262)$, given a cut off value of 684 (expression range $=81-3,840$ ) (Figure 1B).

Knockdown effect of EPHB4 on the functions of gastric cancer cells. The expression of EPHB4 was seen to be different in 
A

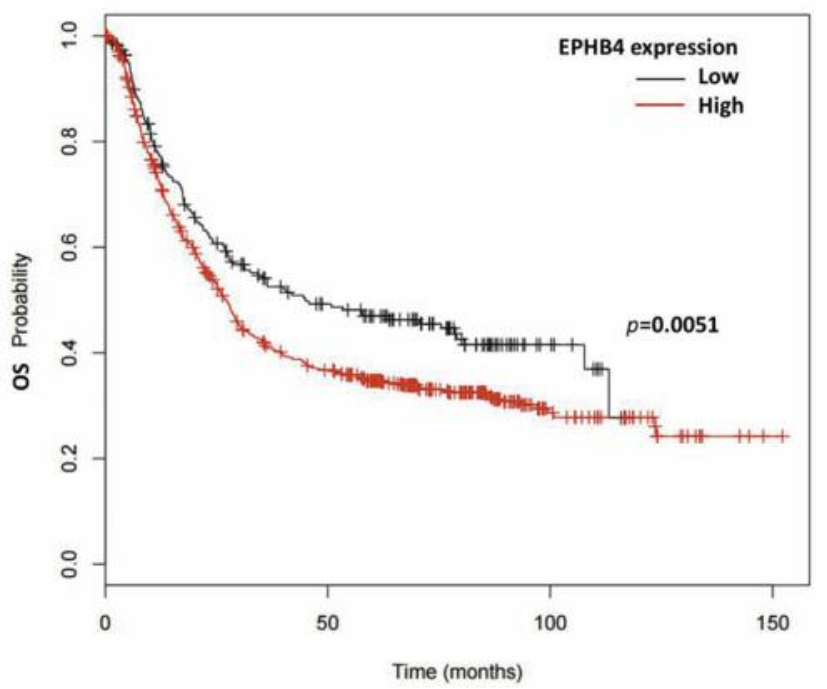

B

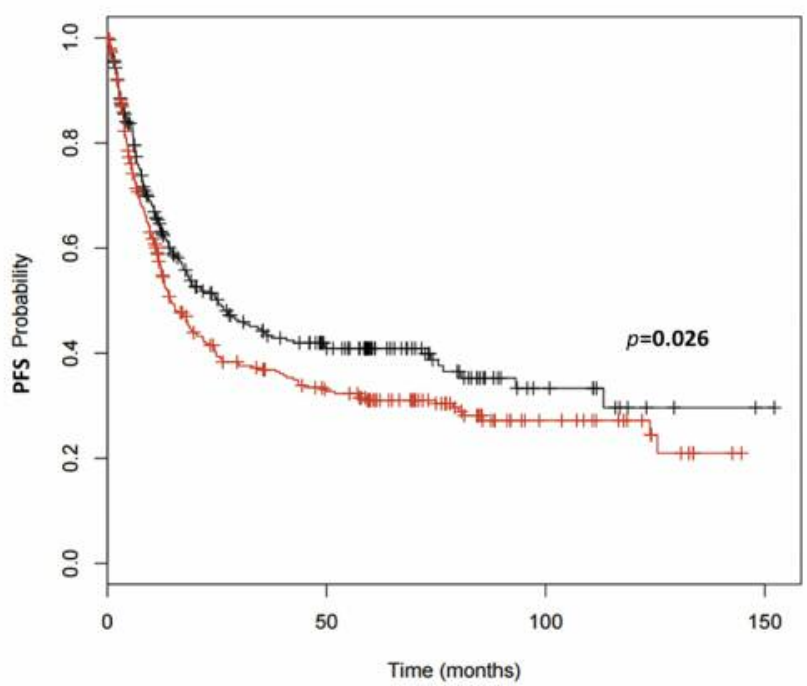

Figure 1. Erythropoietin-producing human hepatoma (EPHB4) expression and survival of patients with gastric cancer. Association between EPHB4 expression and overall (A) and progression-free (B) survival was analyzed using an online Kaplan-Meier survival analysis (KMplot, http://kmplot.com) from 641 cases that were subjected to expression profiling using Affymetrix GeneChip microarray (EPHB4 Probeset ID: 202894_at). Auto-selected cutoff values of 537 and 684 for EPHB4 expression, respectively, were employed in the analysis.

A

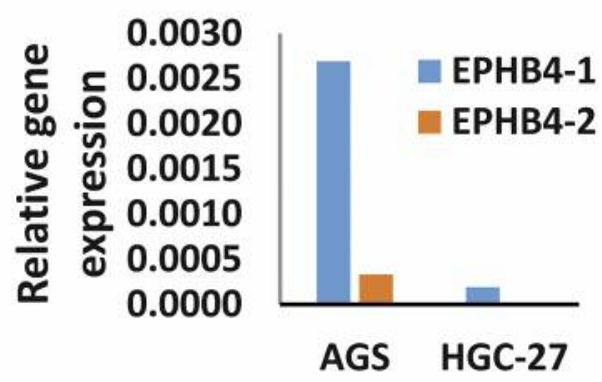

B

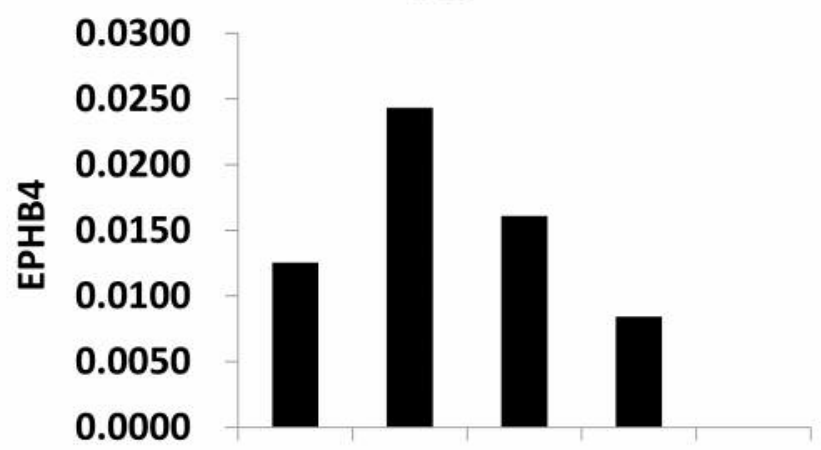

HGC27

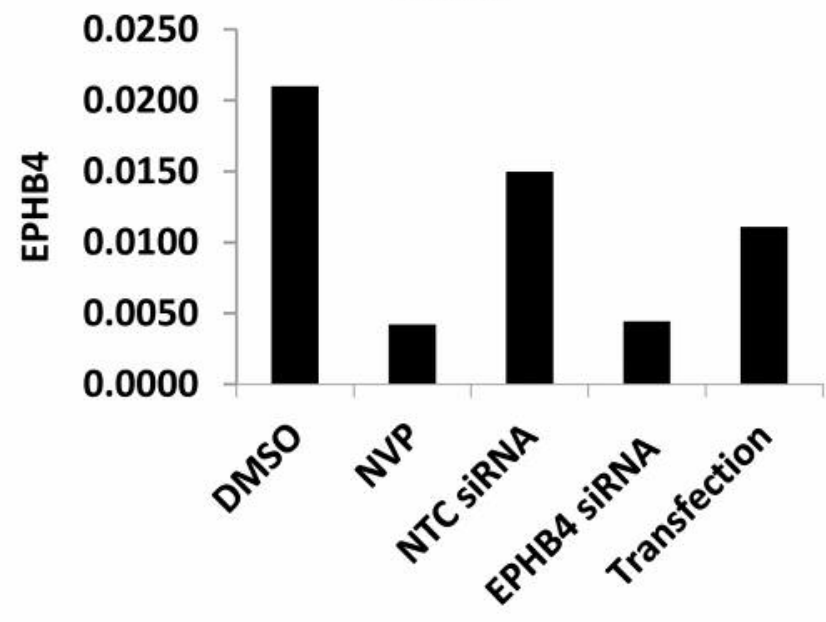

AGS

Figure 2. Erythropoietin-producing human hepatoma (EPHB4) gene expression before and after siRNA silencing in AGS and HGC27 gastric cancer cell lines. A: Differential expression of EPHB4 in the two gastric cancer cell lines as indicated using two sets of EPHB4 primers. $B$ and $C$ : EPHB4 expression after treatment with an EPHB4 kinase inhibitor NVP-BHG712 (NVP) or EPHB4 siRNA in AGS and HGC27 cell lines, respectively. NTC: No template control siRNA; transfection: transfection reagents only. 

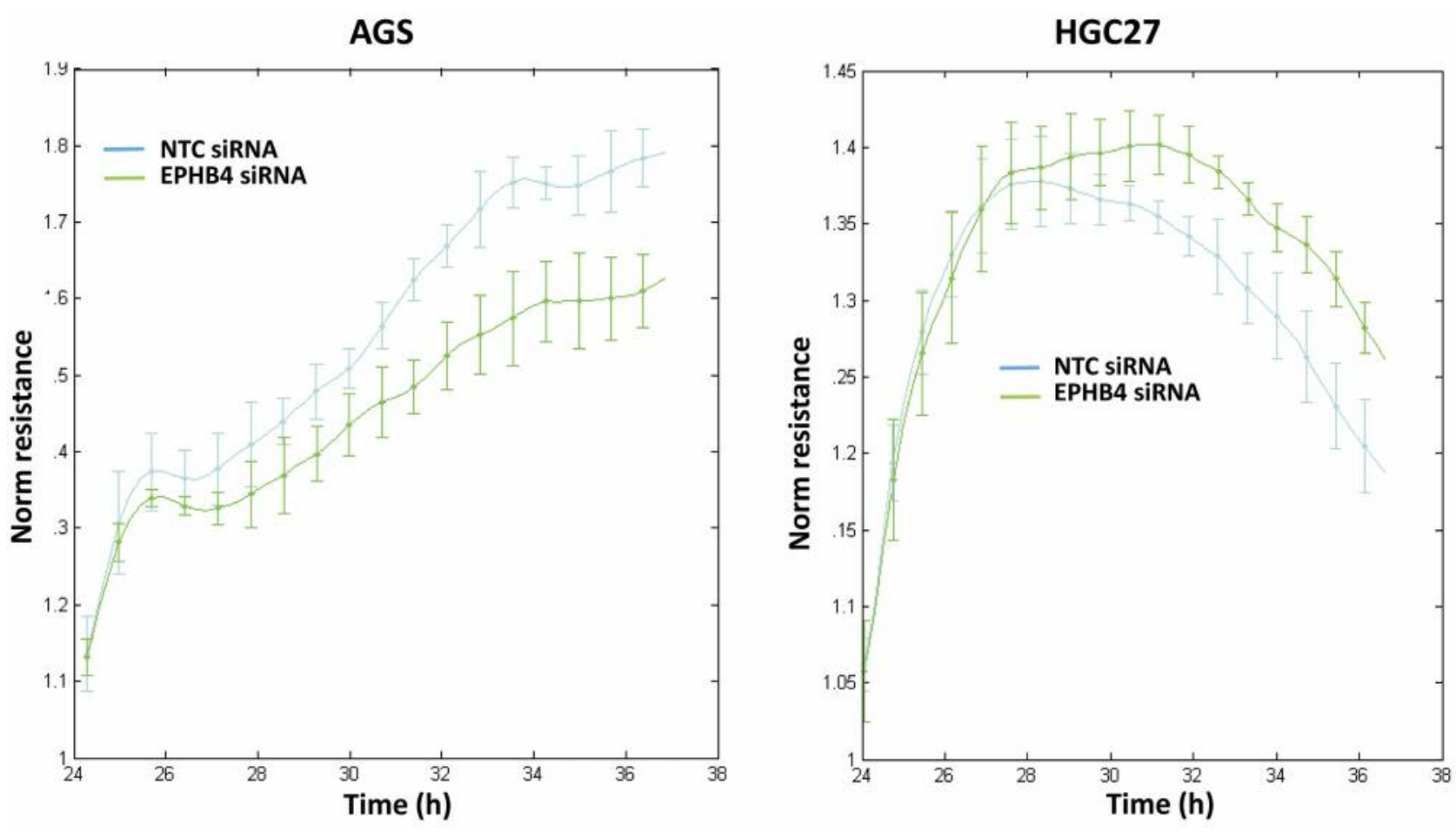

Figure 3. Effect of erythropoietin-producing human hepatoma (EPHB4) knockdown on migration of gastric cancer cells indicated by the Electric Cell-Substrate Impedance Sensing (ECIS) system. AGS and HGC27 cells were seeded onto ECIS 96W1E arrays and adhesion of cells to the culture surface between the two electrodes was monitored via measuring electrical resistance. Once a confluent monolayer had been formed, the cells were damaged by applying electrical current $(2600 \mu \mathrm{A}, 60 \mathrm{kHz})$ for $20 \mathrm{~s}$ to create a break in the cell monolayer. The rate of change in impedance as cells migrated back onto the electrode was subsequently monitored and analyzed. It appeared that EPHB4 knockdown inhibit the migration of AGS cells ( $p<0.05$ vs. no template control (NTC) siRNA but no significance was found for effects in HGC27 cells.

HGC27 and AGS wild-type cells (Figure 2A). We transfected both gastric cell lines using EPHB4 siRNA to knockdown the target gene and NTC siRNA as the control, which was examined using qPCR. The results showed a significantly lower expression of EPHB4 in the knockdown group compared with the control group (2- to 3-fold) (Figure 2B).

Effect of EPHB4 knockdown on ECIS assay. As indicated by the ECIS system (Figure 3), we found that EPHB4 knockdown appeared to reduce post-wound migration of AGS cells compared to the negative siRNA control $(p<0.05)$ (Figure 3A). However, no significant difference in EPHB4 was observed in the HGC cells after EPHB4 silencing (Figure 3B).

Adhesion and proliferation assay. An in vitro matrix adhesion assay was used to investigate the effect of EPHB4 knockdown on the adhesive ability of gastric cancer cell lines. $E P H B 4$ knockdown significantly reduced the adhesion of AGS cells to Matrigel ( $p<0.05 v s$. control) (Figure 4A). However, EPHB4 knockdown significantly increased the adhesive ability of HGC27 cells compared to the control $(p<0.01$; Figure $4 \mathrm{~B})$. In order to determine whether the expression of EPHB4 knockdown affected the growth of gastric cancer cells, we carried out a growth assay. The results did not show any significant effect on growth after EPHB4 silencing compared to the control in both AGS and HGC27 cell lines (Figures 4C and D).

Wound-healing migration assay. In order to determine whether EPHB4 knockdown affected the biological behavior of gastric cancer cell lines, we performed a scratch assay of the cells. EPHB4 knockdown significantly reduced the wound-healing activity compared with the control cells in AGS cells ( $p=0.0056$, Figure 5A). In contrast, knockdown of $E P H B 4$ enhanced the wound-healing potential compared with the control cells in HGC27 cells ( $p=0.0337$, Figure 5B).

\section{Discussion}

Gastric cancer has become the fifth most common cancer worldwide over the past decades. Research indicated that an estimated 4,292,000 new cancer cases and 2814,000 cancer deaths would occur in China in 2015, with gastric cancer being the second most common cancer and the leading cause of 
A

AGS

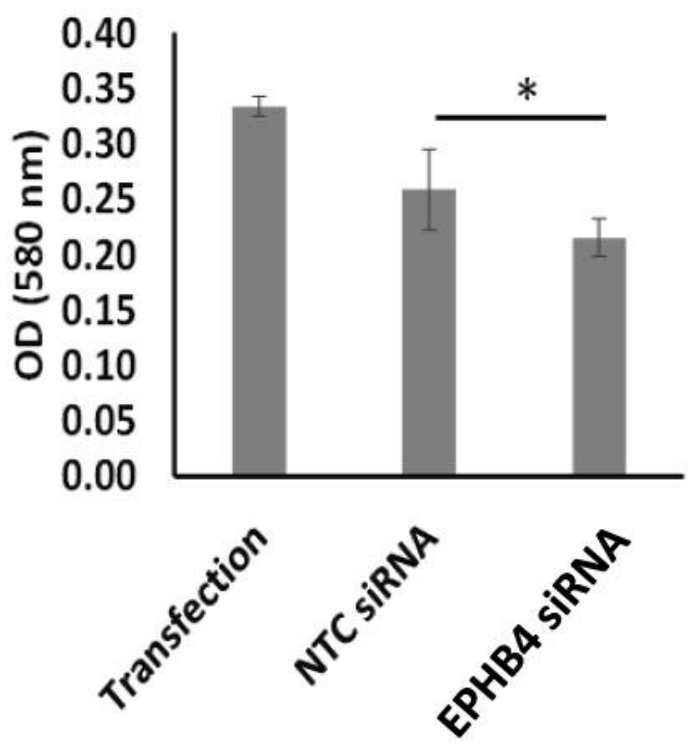

C

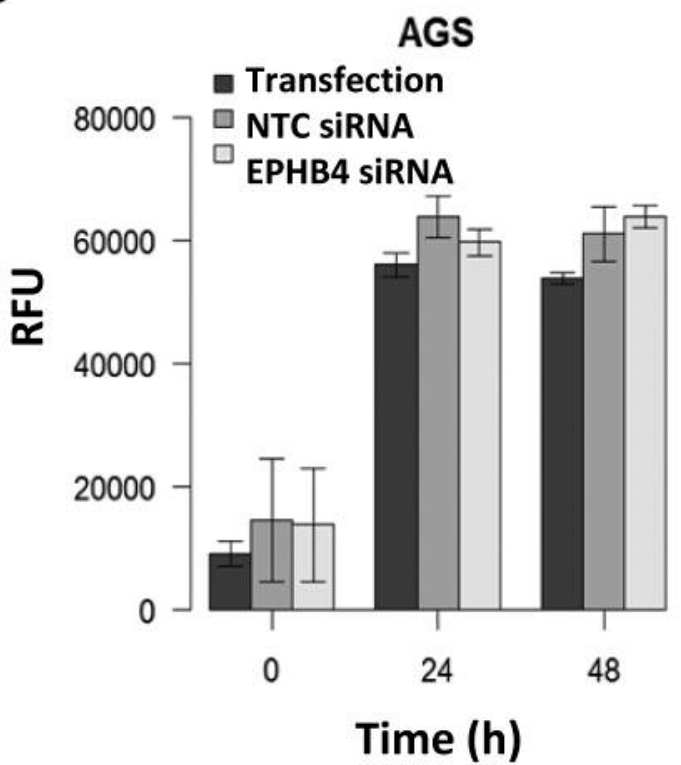

B

\section{HGC27}

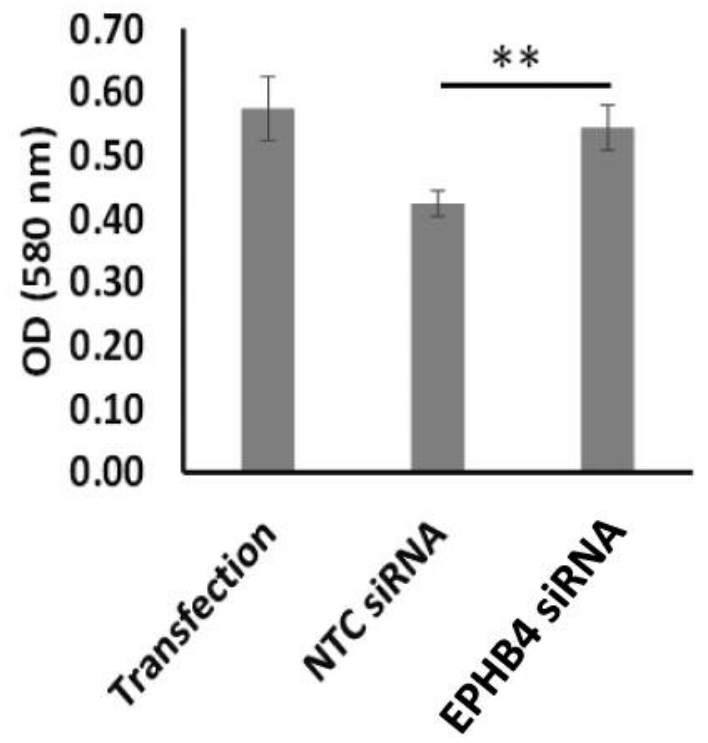

D

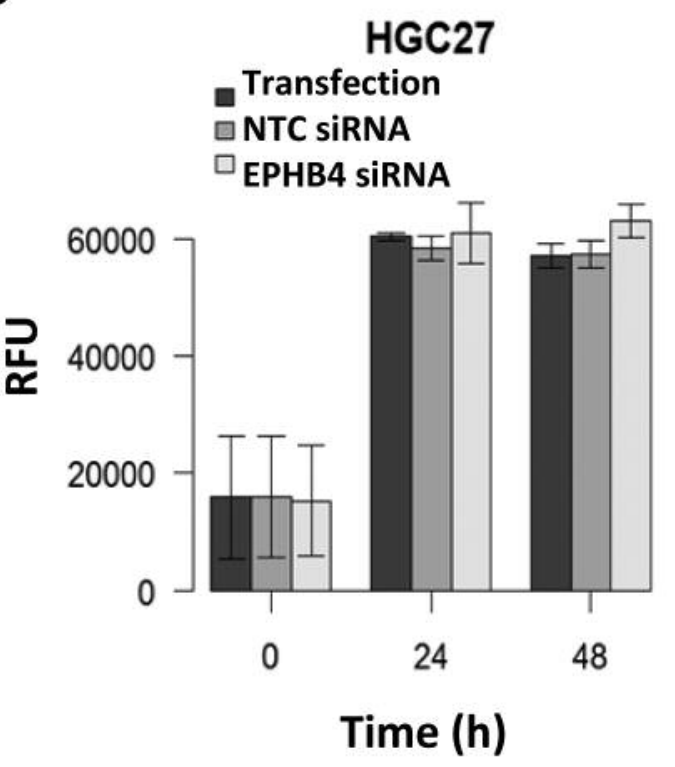

Figure 4. Effect of erythropoietin-producing human hepatoma (EPHB4) silencing on the adhesive $(A, B)$ and proliferative $(C$, D) properties of gastric cancer cells. Adhesion to Matrigel as indicated by crystal violet staining of AGS (A) and HGC27 (B) cells. Proliferation of AGS (C) and HGC27 (D) cells. Significantly different at $* p<0.05$, and $* * p<0.01$.

cancer-related death behind lung cancer. There are $\sim 679,100$ new patients developing this cancer in China each year (14).

EPHB4 is a member of the largest family of RTKs, and was initially isolated from a human hepatocellular carcinoma cell line Hep3Ba (15). Lv et al. found that EPHB4 protein was more highly expressed in colorectal cancer tissues compared with adjacent normal mucosae and EPHB4 acted as a tumor promoter associated with proliferation, invasion, and angiogenesis in colorectal cancer (6). Though the clinical samples of the patients were subjected to PCR and 
A

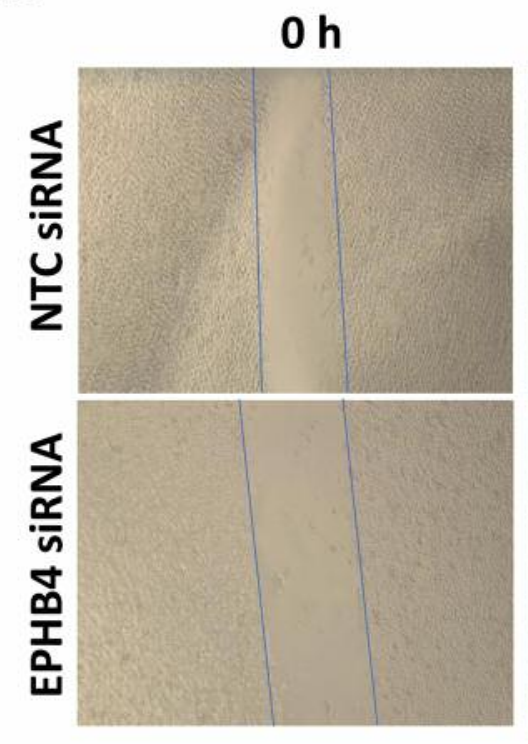

$12 \mathrm{~h}$
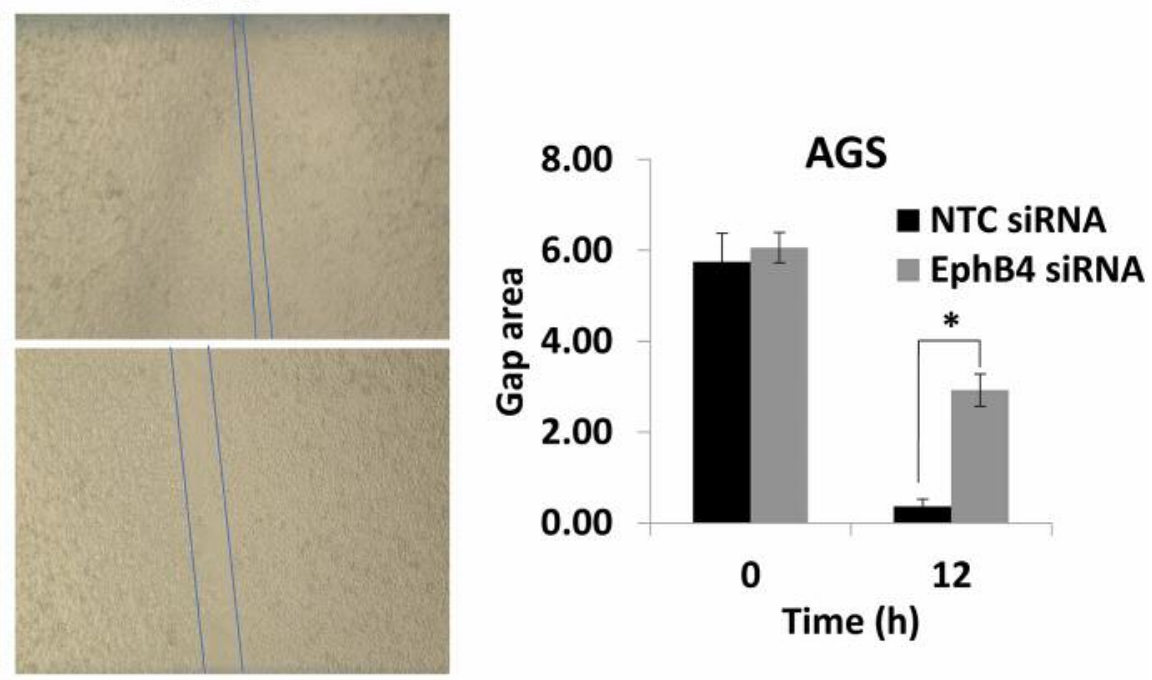

B
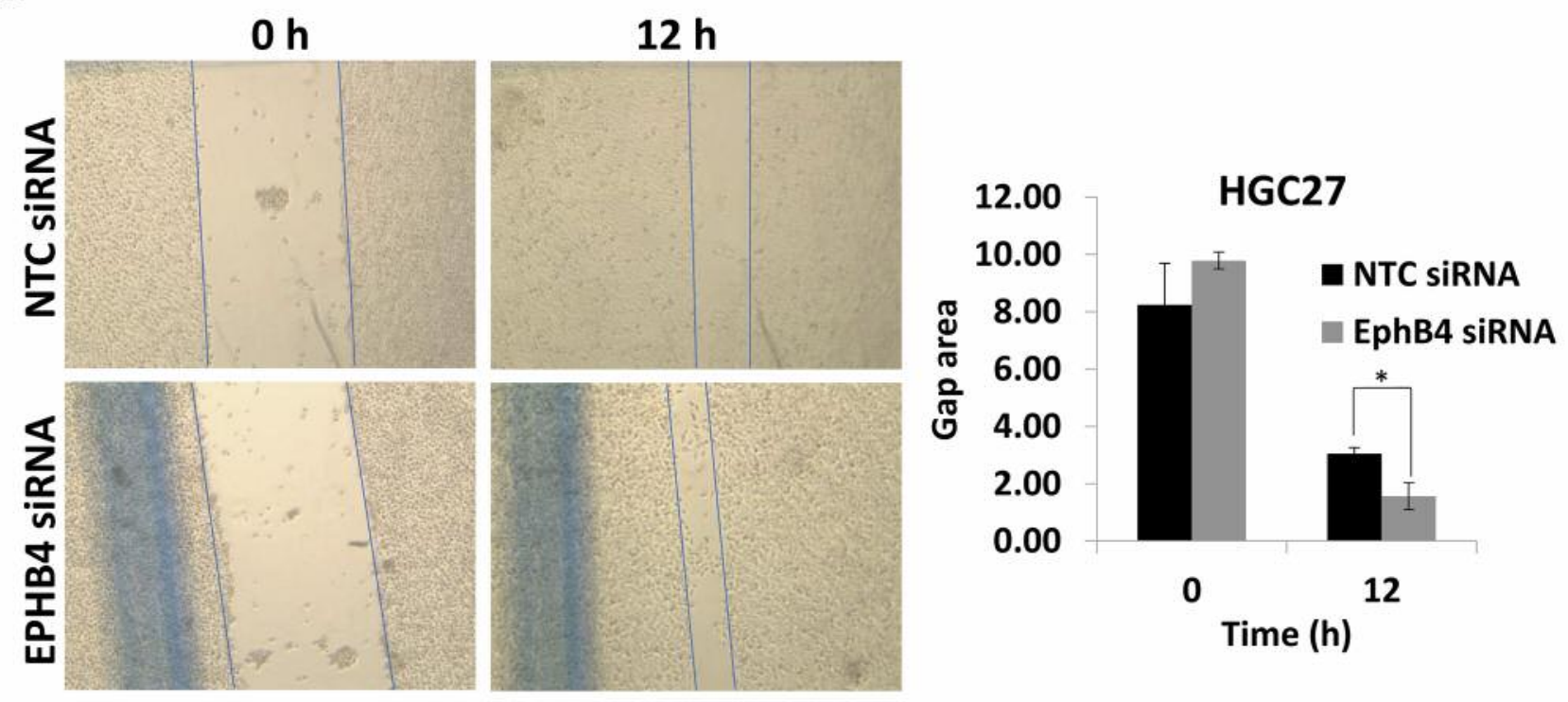

Figure 5. Wound-healing scratch assay was used to assess the effect of erythropoietin-producing human hepatoma (EPHB4) knockdown on the migration of gastric cancer cells. EPHB4 knockdown in AGS cells (A) appeared to reduce wound-healing activity compared to control cells ( $p=0.0056)$, and enhanced wound-healing potential in HGC27 cells $(B)$ compared to control cells $(p=0.0337)$.

western blot studies, Wu et al. showed that high levels of EPHB4 protein expression were associated with poor prognosis in patients with ovarian cancer (15). EPHB4 expression was also significantly higher in soft-tissue sarcomas, and both mRNA and protein expression were markedly increased in synovial sarcoma, while EPHB4 kinase inhibition reduced lung metastasis formation in mouse models (16). Liersch-Lohn et al. reported that overexpression of EPHB4 indicated more advanced and aggressive gastroesophageal junction cancer (8). Huang et al. showed that EPHB4 expression was significantly higher in breast cancer tissues and higher expression of EPHB4 increased tumor proliferation and migration in breast cancer cells (17). High expression of EPHB4 and EPHB2 correlated with poor OS in human epidermal growth factor receptor 2 (HER2)positive breast cancer patients (18). 
In this study, we compared EPHB4 expression in gastric cancer tissues with paired normal mucosal tissue cohort. High EPHB4 expression levels were observed in most colorectal cancer tissues and were significantly higher than that in normal tissue, demonstrating that EPHB4 is overexpressed in gastric cancer. Additional, we found a differentiated expression of EPHB4 in gastric cancer was associated with gender, males exhibited lower expression levels than females $(p=0.0110)$. Research on EPHB4 related to gender and sex hormone levels is rarely reported. However, EPHB2 has been extensively studied in relation to androgen-regulated genes. EPHB2 expression appeared to be temporally and spatially regulated in the developing mouse genital tubercle and differs between sexes, male pattern of expression can be induced in the female genital tubercle by dihydrotestosterone exposure. Dihydrotestosterone exposure alters EPHB2 expression, this indicates that EPHB2 is a candidate androgen-regulated gene (19).

Furthermore, according to tumor location, our study found that gastric tumors expressed higher levels of EPHB4 transcripts in comparison to cardiac tumours $(p<0.001)$. Liersch-Lohn et al. showed that $92 \%$ of gastric carcinomas and $>82 \%$ of esophageal adenocarcinomas overexpressed EPHB4. The analysis of EPHB4 association with staging showed that $100 \%$ of T2-T4 gastric carcinomas and $67 \%$ of T2-T4 esophageal adenocarcinomas overexpressed EPHB4, and expression of $E P H B 4$ in gastric carcinomas were greater than in esophageal adenocarcinomas (46.2\% vs. $22.2 \%)$. Strong and moderate expression of EPHB4 in gastric carcinomas were also greater than in esophageal adenocarcinomas $(77.0 \% \mathrm{vs} .66 .6 \%)$ (8), which our research was consistent with.

We analyzed the association between EPHB4 expression and survival of patients with gastric cancer using an online Kaplan-Meier survival analysis tool with 876 cases and showed that the elevated EPHB4 expression is associated with poor OS and PFS of patients with gastric cancer. Therefore, EPHB 4 can be considered a prognosis indicator in gastric cancer. This is indirectly supported from research in other cancer types. For instance, Li et al. examined the protein expression of EPHB4 and EPHB2 by immunohistochemistry using paraffin-embedded tumor tissues in 111 primary HER2-positive breast cancer tissues and found that high expression of EPHB4 and EPHB2 was correlated with poor OS (18). Higher expression of EPHB4 mRNA in peripheral blood mononuclear cells was also associated with poorer PFS and OS in patients with nonsmall cell lung carcinoma (10). Guijarro-Munoz et al. compared gene-expression profiles in patients with colorectal cancer who responded to bevacizumab treatment with those that did not respond. They found that EPHB4 expression was significantly increased in non-responders, furthermore, high EPHB4 tumor levels were associated with decreased median OS (16 vs. 48 months) (20).
Our data showed that EPHB4 knockdown appeared to reduce wound-healing activity compared with the control AGS cells by the scratch assay, but increased post-wound migration in HGC27 cells compared to the control. Additionally, EPHB4 knockdown significantly increased adhesive ability compared with the control cell line in HGC27 but not AGS cells. The clinical cohort showed the transcript levels of EPHB4 in those with TNM3+4 and $\mathrm{N} 1+2+3$ tumor were significantly higher than the group of those with TNM1+2 and N0 tumors, although this difference was not statistically significant. These results were consistent with our findings in the cell migration capacity of AGS cells, but were contrary to our findings in the HGC27 cells. AGS is a poorly differentiated gastric adenocarcinoma cell line, whereas HGC27 is an undifferentiated gastric cancer cell line. AGS is a p53 wild-type gastric cancer cell line (21), while HGC27 is a p53-mutant cell line (22). p53 is the 'guardian of the genome' and the most commonly mutated tumor suppressor in cancer. p53 activation transcriptionally regulates the expression of its target genes to modulate various cellular processes in response to a wide variety of stress signals, including apoptosis and cell cycle arrest (23). p53 provides a critical barrier to the development of cancer by blocking proliferation or eliminating cancer cells through numerous p53-responsive genes, including in gastric cancer $(24,25)$. In addition, we believe that there must be other causes of the different biological behavior of AGS and HGC27 cell lines, which need to be confirmed by further research.

In conclusion, our research shows that EPHB4 expression is significantly elevated in tumor tissue compared to normal paired tissue in a gastric cancer cohort. An increased EPHB4 expression in gastric cancer correlated with poor OS and PFS. Knockdown of EPHB4 alters adhesive and migratory properties of gastric cancer cells. Taken together, the results indicate that EPHB4 is a potential molecular marker that may be used to predict the prognosis of patients with gastric cancer.

\section{Funding}

This work was supported by the National Natural Science Foundation of China (Grant no. 81541050), the Beijing Talent Training Funding of China (Grant no. 2016000021469G225), and the Beijing Municipal Administration of Hospitals' Youth Programme (Grant no. QML20160105). The authors would like to thank the funding support from CCMRC and Cancer Research Wales as well.

\section{References}

1 Heroult M, Schaffner F and Augustin HG: EPH receptor and ephrin ligand-mediated interactions during angiogenesis and tumor progression. Exp Cell Res 312: 642-650, 2006.

2 Pasquale EB: EPH-ephrin bidirectional signaling in physiology and disease. Cell 133: 38-52, 2008.

3 Xi HQ, Wu XS, Wei B and Chen L: EPH receptors and ephrins as targets for cancer therapy. J Cell Mol Med 16: 2894-2909, 2012. 
4 Gale NW, Holland SJ, Valenzuela DM, Flenniken A, Pan L, Ryan TE, Henkemeyer M, Strebhardt K, Hirai H, Wilkinson DG, Pawson T, Davis S and Yancopoulos GD: EPH receptors and ligands comprise two major specificity subclasses and are reciprocally compartmentalized during embryogenesis. Neuron 17: 9-19, 1996.

5 Li X, Choi WW, Yan R, Yu H, Krasnoperov V, Kumar SR, Schuckman A, Klumpp DJ, Pan CX, Quinn D, Gill IS, Gill PS and Liu R: The differential expression of EPHB2 and EPHB4 receptor kinases in normal bladder and in transitional cell carcinoma of the bladder. PloS One 9: e105326, 2014.

6 Lv J, Xia Q, Wang J, Shen Q, Zhang J and Zhou X: EPHB4 promotes the proliferation, invasion, and angiogenesis of human colorectal cancer. Exp Mol Pathol 100: 402-408, 2016.

7 Mattinzoli D, Rastaldi M, Ikehata M, Armelloni S, Pignatari C, Giardino L, Li M, Alfieri C, Regalia A and Riccardi D: FGF23regulated production of Fetuin-A (AHSG) in osteocytes. Bone 83: 35-47, 2016.

8 Liersch-Lohn B, Slavova N, Buhr HJ and Bennani-Baiti IM: Differential protein expression and oncogenic gene network link tyrosine kinase ephrin B4 receptor to aggressive gastric and gastroesophageal junction cancers. Int J Cancer 138: 1220-1231, 2016.

9 Brantley-Sieders DM, Jiang A, Sarma K, Badu-Nkansah A, Walter DL, Shyr Y and Chen J: EPH/ephrin profiling in human breast cancer reveals significant associations between expression level and clinical outcome. PloS One 6: e24426, 2011.

$10 \mathrm{Yu}$ XM, Wu YC, Liu X, Huang XC, Hou XX, Wang JL, Cheng $\mathrm{XL}$, Mao WM and Ling ZQ: Cell-Free RNA content in peripheral blood as potential biomarkers for detecting circulating tumor cells in non-small cell lung carcinoma. Int J Mol Sci 17: $1845,2016$.

11 Liu R, Ferguson BD, Zhou Y, Naga K, Salgia R, Gill PS and Krasnoperov V: EPHB4 as a therapeutic target in mesothelioma. BMC cancer 13: 269, 2013.

12 Szasz AM, Lanczky A, Nagy A, Forster S, Hark K, Green JE, Boussioutas A, Busuttil R, Szabo A and Gyorffy B: Crossvalidation of survival associated biomarkers in gastric cancer using transcriptomic data of 1,065 patients. Oncotarget 7: 4932249333, 2016.

13 Szász AM, Lánczky A, Nagy Á, Förster S, Hark K, Szabó A and Győrffy B: Cross-validation of survival associated biomarkers in gastric cancer using transcriptomic data of 1,065 patients. Oncotarget: 49322-49333, 2016.

14 Chen W, Zheng R, Baade PD, Zhang S, Zeng H, Bray F, Jemal A, Yu XQ and He J: Cancer statistics in China, 2015. CA Can J Clin 66: 115-132, 2016.

15 Wu Q, Suo Z, Kristensen GB, Baekelandt M and Nesland JM: The prognostic impact of EPHB2/B4 expression on patients with advanced ovarian carcinoma. Gynecol Oncol 102: 15-21, 2006.
16 Becerikli M, Merwart B, Lam MC, Suppelna P, Rittig A, Mirmohammedsadegh A, Stricker I, Theiss C, Singer BB, Jacobsen $F$ and Steinstraesser L: EPHB4 tyrosine-kinase receptor expression and biological significance in soft -tissue sarcoma. Int J Cancer 136: 1781-1791, 2015.

17 Huang G and Li M: The role of EPHB4 and IGF-IR expression in breast cancer cells. Int J Clin Exp Pathol 8: 5997-6004, 2015.

18 Li X, Song C, Huang G, Sun S, Qiao J, Zhao J, Zhao Z and Li $\mathrm{M}$ : The coexpression of EPHB4 and ephrinB2 is associated with poor prognosis in HER2-positive breast cancer. OncoTargets Ther 10: 1735-1742, 2017.

19 Lorenzo AJ, Nguyen MT, Sozubir S, Henkemeyer M and Baker LA: Dihydrotestesterone induction of EPHB2 expression in the female genital tubercle mimics male pattern of expression during embryogenesis. J Urol 170: 1618-1623; discussion 1623, 2003.

20 Guijarro-Munoz I, Sanchez A, Martinez-Martinez E, Garcia JM, Salas C, Provencio M, Alvarez-Vallina L and Sanz L: Gene expression profiling identifies EPHB4 as a potential predictive biomarker in colorectal cancer patients treated with bevacizumab. Med Oncol 30: 572, 2013.

21 Yin Y, Shen Q, Zhang P, Tao R, Chang W, Li R, Xie G, Liu W, Zhang L, Kapoor P, Song S, Ajani J, Mills GB, Chen J, Tao K and Peng G: Chk1 inhibition potentiates the therapeutic efficacy of PARP inhibitor BMN673 in gastric cancer. Am J Cancer Res 7: 473-483, 2017.

22 Forbes S, Clements J, Dawson E, Bamford S, Webb T, Dogan A, Flanagan A, Teague J, Wooster R, Futreal PA and Stratton MR: Cosmic 2005. Br J Cancer 94: 318-322, 2006.

23 Liu J, Zhang C and Feng Z: Tumor suppressor p53 and its gainof-function mutants in cancer. Acta Biochim Biophys Sin 46: 170-179, 2014

24 Wang Y, Xiao H, Wu H, Yao C, He H, Wang C and Li W: G protein subunit alpha q regulates gastric cancer growth via the p53/p21 and MEK/ERK pathways. Oncol Rep 37: 1998-2006, 2017.

25 Mori J, Tanikawa C, Ohnishi N, Funauchi Y, Toyoshima O, Ueda $\mathrm{K}$ and Matsuda K: EPSIN 3, A novel p53 target, regulates the apoptotic pathway and gastric carcinogenesis. Neoplasia 19: 185-195, 2017.
Received June 1, 2017

Revised June 19, 2017

Accepted June 21, 2017 